\title{
A case of testicular atrophy associated with cystic fibrosis
}

\author{
Eriselda Profka1', Giulia Rodari1,2, Federico Giacchetti ${ }^{1,2}$, Alfredo Berrettini3, \\ Gianantonio Manzoni³, Valeria Daccò ${ }^{4}$, Maura Arosio ${ }^{1,2}$, Claudia Giavoli1,2 and \\ Carla Colombo 5
}

1Department of Clinical Sciences and Community Health, University of Milan, Milan, Italy, 2Endocrinology Unit, 3Pediatric Urology Unit, ${ }^{4}$ Cystic Fibrosis Centre, Fondazione IRCCS Ca' Granda Ospedale Maggiore Policlinico, Milan, Italy, and 5Department of Pathophysiology and Transplantation, University of Milan, Fondazione IRCCS Ca' Granda, \section{Ospedale Maggiore Policlinico, Milan, Italy \\ -}

\section{Summary}

An 8-year-old boy with cystic fibrosis came to our attention for an empty scrotum. General physical examination showed a normal penis and hypoplastic scrotum with non-palpable testes bilaterally. Routine blood investigations showed low levels of LH, testosterone, inhibin B and antiMullerian hormone and elevated levels of FSH. Karyotype was normal. An abdominal ultrasound confirmed the absence of the testes into the scrotum, in the inguinal region and abdomen. At laparoscopy were noted bilaterally hypotrophic spermatic vessels, absence of the vas deferens and a closed inner ring. Inguinal exploration found out a small residual testis and histological examination showed fibrotic tissue. This is the first case of testicular atrophy associated to CFTR mutation described. The process that led to bilateral testicular and vas deferens atrophy remains unexplained, a possible influence of CFTR dysfunction cannot be ruled out, although it is possible that these conditions are independently associated.

\section{Learning points:}

- Cystic fibrosis produces a multisystemic disease which can affect also the reproductive tract.

- Nearly $97-98 \%$ of male patients are infertile because of congenital bilateral absence of vas deferens.

- A correlation between cystic fibrosis and bilateral testicular atrophy could be possible.

\section{Background}

Cystic fibrosis (CF) is an autosomal recessive disease resulting from mutations in the CFTR gene with an incidence of 1 every 3500 newborns (1). This disorder produces a multisystemic disease that affects mostly the lungs, pancreas, liver, intestine, sweat glands and the reproductive tract. In fact, infertility is a problem in these patients, especially in males. Nearly $97-98 \%$ of male patients are infertile because of congenital bilateral absence of vas deferens (CBAVD) (1). Such condition may include bilateral absence of vas deferens, atrophy or absence of seminal vesicles and a large portion of epididymis that lead to obstructive azoospermia. Moreover, mutations in CFTR may affect sperm production, maturation and fertilizing abilities as well (1).

Although different cases of infertility reported, to the best of our knowledge has not been so far described of bilateral testicular atrophy associated with cystic fibrosis.

\section{Case presentation}

An 8-year-old boy affected with cystic fibrosis came to our attention for an empty scrotum. The patient, born at 36 weeks, had presented meconium ileus at birth requiring surgical resection; the diagnosis of CF was confirmed by 
high levels of immunoreactive trypsinogen at newborn screening and by genetic test that showed the presence of two CFTR mutations (G542X/H609R, severe genotype). He presented pancreatic insufficiency requiring supplemental pancreatic enzyme therapies. During the childhood, he has experienced numerous episodes of respiratory exacerbation with progressive lung damage. In the medical history, there was a diagnosis of primary not autoimmune hypothyroidism made at the age of 7 years (negative $\mathrm{AbTg}$ and AbTPO and normal thyroid at ultrasound).

General physical examination showed a normal penis, no genital hairs, hypoplastic scrotum with non-palpable testis bilaterally. Height was normal (HT: $121 \mathrm{~cm},-1 \mathrm{SDS}$ ) and BMI low (13 kg/m², $-2.3 \mathrm{SDS})$.

\section{Investigation}

Routine blood investigations showed: LH $0.1 \mathrm{mIU} / \mathrm{mL}$, FSH $24.3 \mathrm{mIU} / \mathrm{mL}$, testosterone $<0.002 \mathrm{ng} / \mathrm{mL}$, inhibin $\mathrm{B}<7 \mathrm{pg} / \mathrm{mL}$, antiMullerian hormone (AMH) $<0.1 \mathrm{ng} / \mathrm{mL}$, prolactin $15.9 \mathrm{ng} / \mathrm{dL}$, TSH $5.3 \mathrm{mIU} / \mathrm{L}(0.28-4.3)$, FT4 16.6 ng/L (8-17), ACTH 13.3 pg/mL, cortisol $12.1 \mu \mathrm{g} / \mathrm{dL}$, IGF-1 $80 \mu \mathrm{g} / \mathrm{L}(-1.59 \mathrm{SDS})$. Hormonal values are outlined in Table 1. Karyotype was normal (46XY).

An abdominal ultrasound was performed and no testes were detected into the scrotum, in the inguinal region or abdomen. Adrenal glands, as well as urinary tract, were normal. Due to the presence of high FSH levels, along with undetectable AMH and inhibin B levels, the hCG test was not performed and the patient was referred to pediatric urologist.

Table 1 Hormonal assessment at diagnosis.

\begin{tabular}{|c|c|c|}
\hline & Results & Reference values \\
\hline $\mathrm{FSH}(\mathrm{mIU} / \mathrm{mL})$ & 24.3 & $1.5-12$ \\
\hline LH (mIU/mL) & 0.1 & $1.7-8.6$ \\
\hline Testosterone (ng/mL) & $<0.002$ & $0.12-0.21$ \\
\hline $\mathrm{AMH}(\mathrm{ng} / \mathrm{mL})$ & $<0.1$ & $1.3-14.8$ \\
\hline Inhibin B (pg/mL) & $<7$ & $5-340$ \\
\hline Prolactin (ng/dL) & 15.9 & $1.7-24$ \\
\hline ACTH (pg/mL) & 13.3 & $0-46$ \\
\hline Cortisol ( $\mu \mathrm{g} / \mathrm{dL})$ & 12.1 & 4.8-19.5 \\
\hline TSH (mIU/L) & 5.3 & $0.28-4.3$ \\
\hline $\mathrm{FT} 4$ (ng/L) & 16.6 & $8-17$ \\
\hline IGF-I ( $\mu \mathrm{g} / \mathrm{L})$ & 80 & $65-225$ \\
\hline
\end{tabular}

$\mathrm{ACTH}$, adrenocorticotropic hormone; $\mathrm{AMH}$, antiMullerian hormone; $\mathrm{FSH}$ follicle-stimulating hormone; fT4, free thyroxine; IGF-1, insulin-like growth factor 1 or somatomedin C; LH, luteinizing hormone; TSH, thyroidstimulating hormone.

\section{Treatment}

After a multidisciplinary discussion, the patient was carefully examined under general anesthesia with laparoscopy approach which noted bilaterally hypotrophic spermatic vessels, absence of the vas deferens and a closed inner ring. An inguinal exploration was performed and a small $(5 \mathrm{~mm})$ residual testis was bilaterally found; histological examination showed fibrotic tissue (Figs 1 and 2).

Thereafter, the patient underwent a regular endocrinological follow-up. At the age of 12.5 years, gonadotropin levels were LH $37.7 \mathrm{mUI} / \mathrm{mL}$ and FSH 83 $\mathrm{mUI} / \mathrm{mL}$ while testosterone was $<0.002 \mathrm{ng} / \mathrm{mL}$. At this age, therapy with testosterone esters was started, to induce puberty.

\section{Outcome and follow-up}

Currently, patient is 17 years old and presents chronic respiratory infection with Pseudomonas aeruginosa, a severe respiratory impairment for which he was registered on the lung transplant list. The boy is continuing his testosterone replacement therapy. On our last physical examination, he had normal secondary sexual characteristics.

\section{Discussion}

This is the first report of bilateral testicular atrophy associated with cystic fibrosis. The presence of external male genitalia suggests that a process of gonad atrophy

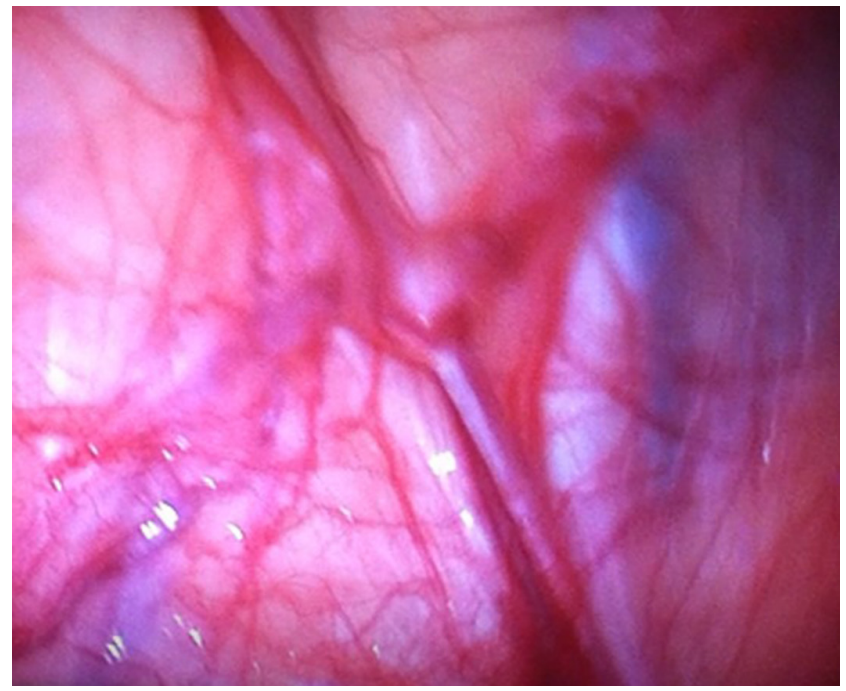

Figure 1

Transabdominal laparoscopy shows absent testis with atrophic vessels and absent vas. 


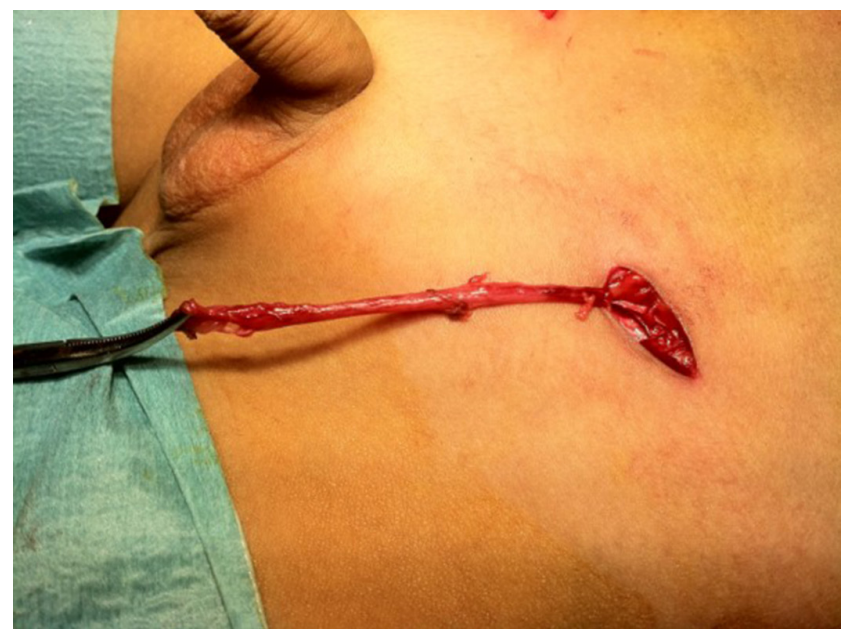

Figure 2

Bilateral inguinal exploration confirms absent testis and atrophic vessels.

had occurred in late pregnancy or soon after birth, since the presence of functional testis is fundamental for its development. The laparoscopic finding of small and fibrotic residual testes also supports this hypothesis. In fact, it is known that the development of testis during embryogenesis is directed by the presence of the $\mathrm{Y}$ chromosome, especially from the presence of the SRY region (2). Under the influence of SRY, primordial germ cells become surrounded by primitive Sertoli cells to form seminiferous or sex cords that develop into seminiferous tubules and from the 8th week of gestation Leydig cells begin to form. Then, under the influence of maternal human chorionic gonadotropin (hCG) initially and later of LH and FSH from the fetal pituitary gland, immature Leydig cells, Sertoli cells, and germ cells undergo differentiation, proliferation, and organization. Testosterone production from fetal Leydig cells increases progressively and induces development of the epididymis, vas deferens, and seminal vesicles from wolffian or mesonephric ducts. Conversion of testosterone to $5 \alpha$-dihydrotestosterone in the urogenital tract leads to the formation of the prostate from the urogenital sinus, the penis from the genital tubercles and folds, and the scrotum from the urogenital swelling. In the absence of testosterone production or action, internal and external female genitalia develop. AMH secretion from the fetal Sertoli cells causes regression of the müllerian or paramesonephric ducts and prevents the formation of a uterus and fallopian tubes (2). Testicular descent is a complex process involving genetic, anatomical, hormonal and environmental factors and conditions affecting any of this factors can cause cryptorchidism (3). Previous authors have described cases of cryptorchidism associated with CF (4); Differentiating between cryptorchidism and testicular atrophy is a diagnostic challenge. In a very recent paper by Jespersen and colleague, AMH and inhibin B from birth to 15 years of age and FSH during first year of life at certain cut-off levels appeared to be excellent diagnostic markers of functional testicular and for differentiating cryptorchidism and anorchia. In particular, AMH and inhibin B concentrations resulted to be significantly lower in anorchid boys (5). Unfortunately, we did not know FSH levels during the first year of life, but the low levels of inhibin B and AMH found in our patient is suggestive for gonad atrophy.

This is the first case of unexplained testicular atrophy associated with CFTR mutation described. It is not known if these conditions are related to each other or if it is simply a random independent association.

It is known that approximately $97-98 \%$ of male patients with CF are infertile due to congenital bilateral absence of vas deferens which leads to azoospermia. CFTR is expressed in the epididymis and vas deferent since early gestation, suggesting an important role during development of these structures (6). It has been proposed that atrophy and involution of male excurrent ductal system may be caused by intrauterine obstruction by dehydrated secretions (6). In fact, Marcorelles and colleagues have documented that in cystic fibrosis fetuses there were no excretory ducts agenesis or obstruction until 22 WG; in addition, a focal inflammatory pattern in oldest cases suggested a disruptive mechanism (7). CFTR is also implicated in other important mechanisms. It seems to play a role in mediating the effect of FSH on spermatogenesis, in regulating junctional complexes and blood barrier within the testes, and may influence various stages of spermatogenesis and sperm capacitation (1).

The present case is peculiar, since unexplained testicular atrophy was documented for the first time. Since it was not possible to collect data on past history (pregnancy, birth and first years of life), it is difficult to assess the exact time in which the process of bilateral testicular atrophy began and therefore to understand its pathogenesis. Indeed, FSH levels, though high for age, were not markedly elevated. Thus, one may hypothesize that the child had the normal mini-puberty phase, as expected from birth till the 6th month of life, with the rising of testicular inhibin B preventing a marked FSH increase. Similarly, an eventual testosterone production during the same period might have prevented precocious LH rise. The process that led to bilateral testicular and vas deferens atrophy remains unexplained. It is possible that 
these conditions are independently associated. However, a possible influence of CFTR dysfunction cannot be ruled out. Indeed, it is tempting to speculate that testicular and excretory ducts atrophy might share the same physiopathological destructive mechanism (7). Moreover, the above-described role of CTFR within the testes (1) tends to support the hypothesis of a link between the two conditions.

\section{Declaration of interest}

The authors declare that there is no conflict of interest that could be perceived as prejudicing the impartiality of the research reported.

\section{Funding}

This research did not receive any specific grant from any funding agency in the public, commercial or not-for-profit sector.

\section{Patient consent}

Written informed consent has been obtained from the patient's father for publication of the submitted article and accompanying images.

\section{Author contribution statement}

E P and C G wrote the manuscript. E P, G R, F G, V D and C G performed patient follow up. A B and $G M$ performed urologic follow up including laparoscopy approach. C C and M A revised critically the manuscript. All authors contributed to manuscript revision, read and approved the submitted version.

\section{References}

1 Chen H, Ruan YC, Xu WM, Chen J \& Chan HC. Regulation of male fertility by CFTR and implications in male infertility. Human Reproduction Update 201218 703-713. (https://doi.org/10.1093/ humupd/dms027)

2 Yasmin S. Development of genital ducts and external genitalia in the early human embryo. Journal of Obstetrics and Gynaecology Research 201036 929-937. (https://doi.org/10.1111/j.14470756.2010.01272.x)

3 Rodprasert W, Virtanen HE, Mäkelä JA \& Toppari J. Hypogonadism and cryptorchidism. Frontiers in Endocrinology 201910 906. (https:// doi.org/10.3389/fendo.2019.00906)

4 Goshen R, Kerem E, Shoshani T, Kerem BS, Feigin E, Zamir O \& Yahav Y. Cystic fibrosis manifested as undescended testis and absence of vas deferens. Pediatrics $199290982-983$.

5 Jespersen K, Ljubicic ML, Johannsen TH, Christiansen $\mathrm{P}$, Skakkebaek NE \& Juul A. Distinguishing between hidden testes and anorchia: the role of endocrine evaluation in infancy and childhood. European Journal of Endocrinology 2020183 107-117. (https://doi. org/10.1530/EJE-20-0041)

6 Tizzano EF, Silver MM, Chitayat D, Benichou JC \& Buchwald M. Differential cellular expressione of cystic fibrosis trans membrane regulator in human reproductive tissues. American Journal of Pathology 1994144 906-914.

7 Marcorelles P, Gillet D, Friocourt G, Ledè F, Samaison L, Huguen G $\&$ Ferec C. Cystic fibrosis transmembrane conductance regulator protein expression in the male excretory duct system during development. Human Pathology 201243 390-397. (https://doi. org/10.1016/j.humpath.2011.04.031)

Received in final form 30 July 2020

Accepted 25 August 2020 